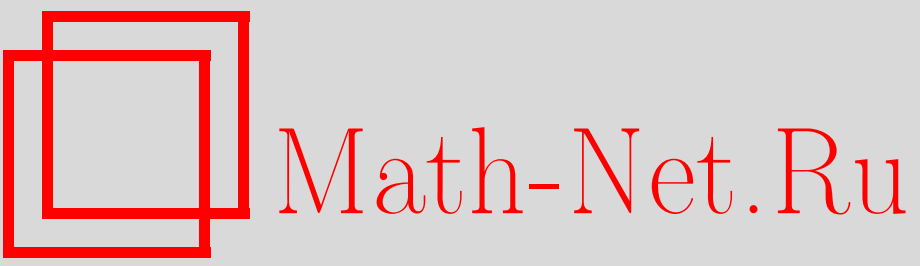

Д. Ф. Дигор, В. А. Москаленко, Представление Ванье для трехзонной модели Хаббарда, ТМФ, 2002, том 130, номер 2, 320-338

DOI: https://doi.org/10.4213/tmf305

Использование Общероссийского математического портала Math-Net.Ru подразумевает, что вы прочитали и согласны с пользовательским соглашением

http://www.mathnet.ru/rus/agreement

Параметры загрузки:

IP: 54.198 .67 .100

26 апреля 2023 г., 18:13:33 
ТЕОРЕТИЧЕСКАЯ

И МАТЕМАТИЧЕСКАЯ

ФИЗИКА

Том 130, № 2

февраль, 2002

(C) 2002 г.

Д. Ф. Дигор* , В. А. Москаленко ${ }^{\dagger}$

\section{ПРЕДСТАВЛЕНИЕ ВАНЬЕ ДЛЯ ТРЕХЗОННОЙ МОДЕЛИ ХАББАРДА}

Получено ячеечное представление гамильтониана $(d-p)$-модели. Использованы ортогонализованные на узлах решетки меди орбитали Ванье для дырок, принадлежащих ионам меди и кислорода. Исходным этапом вычислений является диагонализация кинетической энергии кислородных дырок и введение на этой основе двух диагонализуюших орбиталей кислородных фермионов. Эти две последние моды обладают существенно различными локальными энергиями, что заметно сказывается на результатах теории. Полученный гамильтониан представлен в виде суммы главного локального вклада и возмущения, определяющего делокализацию ванье-фермионов. Определены низколежащие состояния локального гамильтониана и их энергетический спектр. Показано, что введение диагонализующих фермионов приводит к существенному понижению энергии синглета Жанга-Райса.

\section{1. ВВЕДЕНИЕ}

Со времени открытия высокотемпературной сверхпроводимости велись усиленные поиски теоретических моделей, которые могли бы ее описать. Наиболее известной особенностью высокотемпературных материалов, которая считается ответственной за явление сверхпроводимости, является наличие слоистой структуры, содержашей $\mathrm{CuO}_{2}$ плоскости. Именно в данных плоскостях в основном и протекает это явление. Модельньй гамильтониан, описывающий движение носителей заряда в этих плоскостях, был предложен в работах [1]-[3]. В элементарной ячейке $\mathrm{CuO}_{2}$-плоскости содержится только одна $d_{x^{2}-y^{2}}$-орбиталь меди и по одной $p$-орбитали на двух атомах кислорода,

$$
p^{x}\left(\vec{l}=\vec{i} \pm \frac{a}{2} \hat{x}\right), \quad p^{y}\left(\vec{l}=\vec{i} \pm \frac{a}{2} \hat{y}\right)
$$

Орбитали меди локализованы на узлах $\vec{i}$ квадратной решетки меди с постоянной $a$ и базисными векторами $\hat{x}$ и $\hat{y}$. Орбитали кислорода локализованы на узлах $\vec{l}$. Ион $\mathrm{Cu}^{1+}$

* Институт прикладной физики АН Республики Молдова, Кишинев, Молдова. E-mail: statphys@asm.md

† Объединенный институт ядерных исследований, Дубна, Московская обл., Россия. E-mail: moskalen@thsun1.jinr.ru 
$\left(3 d^{10}\right.$-состояние) отвечает полностью заполненным $d$-орбиталям, а ион $\mathrm{O}^{2-}\left(2 p^{6}\right.$-состояние) - полностью заполненным $p$-орбиталям. Ниже используется дырочное представление с вакуумным состоянием, отвечающим электронному состоянию $3 d^{10} 2 p^{6}$.

Гамильтониан $(d-p)$-модели принимает вид

$$
H=H_{d}+H_{p}+H_{d-p}
$$

где

$$
\begin{aligned}
H_{d} & =\epsilon_{d} \sum_{i, \sigma} n_{i \sigma}^{d}+U_{d} \sum_{i} n_{i \uparrow}^{d} n_{i \downarrow}^{d}, \\
H_{p} & =\epsilon_{p} \sum_{l, \sigma} n_{l \sigma}^{p}+t_{p p} \sum_{\left\langle l, l^{\prime}\right\rangle, \sigma} p_{l, \sigma}^{+} p_{l^{\prime}, \sigma} S_{l l^{\prime}}+U_{p} \sum_{l} n_{l \uparrow}^{p} n_{l \downarrow}^{p}, \\
H_{d-p} & =t_{d p} \sum_{\langle i, l\rangle, \sigma}\left(d_{i, \sigma}^{+} p_{l, \sigma}+\text { h.c. }\right) S_{i l}+U_{d p} \sum_{\langle i, l\rangle} n_{i}^{d} n_{l}^{p} .
\end{aligned}
$$

Здесь $d_{i, \sigma}\left(d_{i, \sigma}^{+}\right)$и $p_{l, \sigma}\left(p_{l, \sigma}^{+}\right)$- операторы уничтожения (рождения) соответственно $d$ - и $p$-дырок, расположенных на узлах меди $i$ и кислорода $l$ и обладающих спином $\sigma$. Плотности числа дырок $n_{i \sigma}^{d}=d_{i, \sigma}^{+} d_{i, \sigma}, n_{i}^{d}=n_{i \uparrow}^{d}+n_{i \downarrow}^{d}, n_{l \sigma}^{p}=p_{l, \sigma}^{+} p_{l, \sigma}, n_{l}^{p}=n_{l \uparrow}^{p}+n_{l \downarrow}^{p}$. Через $\epsilon_{d}$ и $\epsilon_{p}$ обозначены одноузельные энергии дырок на соответствующих ионах, отсчитанные от химического потенциала системы, $\epsilon_{\alpha}=\bar{\epsilon}_{\alpha}-\mu(\alpha=d, p), U_{d}$ и $U_{p}$ - энергии кулоновского отталкивания дырок на узлах меди и кислорода, соответственно, $U_{d p}-$ энергия межузельного отталкивания. Интегралы гибридизации состояний $t_{d p}$ и $t_{p p}$ умножаются на специально введенные фазовые факторы $S_{i l}$ и $S_{l l^{\prime}}$. Выбор этих факторов для двух соседних орбиталей соответствует выбранному нами распределению знаковых функций самих орбиталей. Здесь мы следуем работам [4]-[13]. С учетом выбранного нами фазового фактора $S_{i l}$ можно переписать вклад в $(2)$ от $(d-p)$-гибридизации в виде взаимодействия $d_{i, \sigma}$-орбитали меди с кислородным орбитальным кластером $\beta_{i, \sigma}$, составленным из дырок четырех ионов кислорода, окружающих центральный ион меди с индексом узла $i$,

$$
2 t_{d p} \sum_{i, \sigma}\left(d_{i, \sigma}^{+} \beta_{i, \sigma}+\beta_{i, \sigma}^{+} d_{i, \sigma}\right)
$$

где оператор уничтожения кластера $\beta_{i, \sigma}$ имеет вид

$$
\beta_{i, \sigma}=\frac{1}{2}\left(p_{\vec{i}+\frac{a}{2} \hat{x}, \sigma}^{x}-p_{\vec{i}+\frac{a}{2} \hat{y}, \sigma}^{y}-p_{\vec{i}-\frac{a}{2} \hat{x}, \sigma}^{x}+p_{\vec{i}-\frac{a}{2} \hat{y}, \sigma}^{y}\right) .
$$

Эта орбиталь введена в работе [4], где было отмечено ее значение для образования локальных синглетного и триплетного состояний дырки на меди и допированных дырок на кислороде. Было показано, что этот локальньй синглет, образованный дыркой на центральном ионе $\mathrm{Cu}^{2+}$ и дыркой на четырех окружающих атомах кислорода, имеет значительную энергию связи и движется по решетке подобно дыркам в эффективной однозонной модели Хаббарда. Авторы [4] высказали предположение о возможности описания низкоэнергетических свойств $(d-p)$-модели на основе ее редукции к более простой

6 Теоретическая и математическая физика, т. 130, № 2, 2002 г. 
эффективной однозонной модели подобно тому, как однозонная модель Хаббарда была сведена Андерсоном [14] к так называемой $(t-J)$-модели. Данная концепция обсуждалась в работах [5]-[13] и получила свое дальнейшее развитие в работах [15]-[43].

Наш подход к данной проблеме отличается от подхода, содержашегося в цитированных выше работах. Вместо введения с самого начала кластерной орбитали (4) и связанных с ней величин мы вводим прежде всего новые кислородные орбитали, которые диагонализуют кинетическую энергию кислородных дырок. Лишь затем на этой новой основе рассматривается процесс образования связанных состояний и изучаются динамические свойства трехзонной модели. При этом удается учесть роль такой важной для теории величины, как энергия переноса дырок с ионов меди на ионы кислорода при допировании вешества.

\section{2. ДИАГОНАЛИЗУЮЩИЕ ОРБИТАЛИ}

Вернемся к гамильтониану (1) и рассмотрим ту его часть $H_{p}^{0}$, которая связана с движением кислородных дырок,

$$
H_{p}^{0}=\epsilon_{p} \sum_{l, \sigma} p_{l, \sigma}^{+} p_{l, \sigma}+t_{p p} \sum_{\left\langle l, l^{\prime}\right\rangle, \sigma} p_{l, \sigma}^{+} p_{l^{\prime}, \sigma} S_{l, l^{\prime}}
$$

Последнее слагаемое в (5), обязанное кислород-кислородной гибридизации состояний дырок, с учетом выбранных нами фазовых факторов приобретает вид

$$
\begin{aligned}
& t_{p p} \sum_{\left\langle l, l^{\prime}\right\rangle, \sigma} p_{l, \sigma}^{+} p_{l^{\prime}, \sigma} S_{l, l^{\prime}}=\frac{1}{2} \sum_{l, \sigma}\left\{p _ { \vec { i } + \frac { a } { 2 } \hat { x } , \sigma } ^ { x + } \left[-p_{\vec{i}+a \hat{x}+\frac{a}{2} \hat{y}, \sigma}^{y}+\right.\right. \\
& \left.\quad+p_{\vec{i}+\frac{a}{2} \hat{y}, \sigma}^{y}-p_{\vec{i}-\frac{a}{2} \hat{y}, \sigma}^{y}+p_{\vec{i}+a \hat{x}-\frac{a}{2} \hat{y}, \sigma}^{y}\right]+ \\
& \quad+p_{\vec{i}+\frac{a}{2} \hat{y}, \sigma}^{y+}\left[-p_{\vec{i}+\frac{a}{2} \hat{x}+a \hat{y}, \sigma}^{x}+p_{\vec{i}-\frac{a}{2} \hat{x}+a \hat{y}, \sigma}^{x}-p_{\vec{i}-\frac{a}{2} \hat{x}, \sigma}^{x}+p_{\vec{i}+\frac{a}{2} \hat{x}, \sigma}^{x}\right]+ \\
& \quad+p_{\vec{i}-\frac{a}{2} \hat{x}, \sigma}^{x+}\left[-p_{\vec{i}+\frac{a}{2} \hat{y}, \sigma}^{y}+p_{\vec{i}-a \hat{x}+\frac{a}{2} \hat{y}, \sigma}^{y}-p_{\vec{i}-a \hat{x}-\frac{a}{2} \hat{y}, \sigma}^{y}+p_{\vec{i}-\frac{a}{2} \hat{y}, \sigma}^{y}\right]+ \\
& \left.\quad+p_{\vec{i}-\frac{a}{2} \hat{x}, \sigma}^{y+}\left[-p_{\vec{i}+\frac{a}{2} \hat{x}, \sigma}^{x}+p_{\vec{i}-\frac{a}{2} \hat{x}, \sigma}^{x}-p_{\vec{i}-\frac{a}{2} \hat{x}-a \hat{y}, \sigma}^{x}+p_{\vec{i}+\frac{a}{2} \hat{x}-a \hat{y}, \sigma}^{x}\right]\right\}
\end{aligned}
$$

Перейдем к фурье-представлению для дырочных операторов $p^{x}$ и $p^{y}$, используя пространство волновых векторов $\vec{k}$ зоны Бриллюэна решетки лишш одних ионов меди,

$$
\begin{aligned}
& p_{\vec{i} \pm \frac{a}{2} \hat{x}, \sigma}^{x}=\frac{1}{\sqrt{N}} \sum_{\vec{k}} e^{-i \vec{k}\left(\vec{R}_{i \pm \frac{a}{2} \hat{x}}\right)} p_{\vec{k}, \sigma}^{x}, \\
& p_{\vec{i} \pm \frac{a}{2} \hat{y}, \sigma}^{y}=\frac{1}{\sqrt{N}} \sum_{\vec{k}} e^{-i \vec{k}\left(\vec{R}_{i \pm \frac{a}{2} \hat{y}}\right)} p_{\vec{k}, \sigma}^{y} .
\end{aligned}
$$


Здесь $N$ - число ионов решетки меди. Компоненты волнового вектора $\vec{k}=\left(k_{x}, k_{y}\right)$ меняются в пределах от $-\pi / a$ до $\pi / a$, где $a$ - постоянная решетки. $\mathrm{B} \vec{k}$-представлении оператор орбитали кислородного кластера (4)

$$
\beta_{i, \sigma}=\frac{i}{\sqrt{N}} \sum_{\vec{k}} e^{-i \vec{k} \vec{R}_{i}}\left(-p_{\vec{k}, \sigma}^{x} S_{x}(\vec{k})+p_{\vec{k}, \sigma}^{y} S_{y}(\vec{k})\right)
$$

где $S_{x}(\vec{k})=\sin \left(k_{x} a / 2\right)$ и $S_{y}(\vec{k})=\sin \left(k_{y} a / 2\right)$. В представлении волновых векторов оба слагаемых энергии (5) кислородных дырок имеют вид

$$
\begin{aligned}
\epsilon_{p} \sum_{l, \sigma} p_{l, \sigma}^{+} p_{l, \sigma} & =\epsilon_{p} \sum_{\vec{k}, \sigma}\left(p_{\vec{k}, \sigma}^{x+} p_{\vec{k}, \sigma}^{x}+p_{\vec{k}, \sigma}^{y+} p_{\vec{k}, \sigma}^{y}\right) \\
t_{p p} \sum_{\left\langle l, l^{\prime}\right\rangle, \sigma} p_{l, \sigma}^{+} p_{l^{\prime}, \sigma} S_{l, l^{\prime}} & =4 t_{p p} \sum_{\vec{k}, \sigma} S_{x} S_{y}\left(p_{\vec{k}, \sigma}^{x+} p_{\vec{k}, \sigma}^{y}+p_{\vec{k}, \sigma}^{y+} p_{\vec{k}, \sigma}^{x}\right) .
\end{aligned}
$$

Каноническое преобразование, которое диагонализует эти слагаемые, имеет вид

$$
\begin{aligned}
& p_{\vec{k}, \sigma}^{x}=u(\vec{k}) a_{\vec{k}, \sigma}+v(\vec{k}) b_{\vec{k}, \sigma} \\
& p_{\vec{k}, \sigma}^{y}=-v^{*}(\vec{k}) a_{\vec{k}, \sigma}+u^{*}(\vec{k}) b_{\vec{k}, \sigma} .
\end{aligned}
$$

Коэффицциенты преобразования (11) подчиним условиям

$$
|u(\vec{k})|^{2}+|v(\vec{k})|^{2}=1, \quad\left(u^{*}(\vec{k})\right)^{2}=(v(\vec{k}))^{2} .
$$

Второе условие (12) следует из необходимости диагонализации оператора (10). Фактически оба слагаемых в (5) диагонализуются независимо. Тем самым коэффициенты преобразования не зависят от величины энергии перескока $t_{p p}$. В новых амплитудах $a_{\vec{k}, \sigma}$ и $b_{\vec{k}, \sigma}$ оператор кинетической энергии принимает вид

$$
\begin{aligned}
H_{p}^{0}= & \sum_{\vec{k}, \sigma}\left\{\epsilon_{p}\left(a_{\vec{k}, \sigma}^{+} a_{\vec{k}, \sigma}+b_{\vec{k}, \sigma}^{+} b_{\vec{k}, \sigma}\right)+\right. \\
& \left.+4 t_{p p} S_{x} S_{y}\left(u(\vec{k}) v(\vec{k})+u^{*}(\vec{k}) v^{*}(\vec{k})\right)\left(b_{\vec{k}, \sigma}^{+} b_{\vec{k}, \sigma}-a_{\vec{k}, \sigma}^{+} a_{\vec{k}, \sigma}\right)\right\} .
\end{aligned}
$$

Из условий (12) следует равенство модулей коэффицциентов преобразования $|u(\vec{k})|=$ $|v(\vec{k})|=1 / \sqrt{2}$, однако сохраняется произвол в выборе их фазовых множителей. Уменьшая этот произвол, потребуем, чтобы при $t_{p p}>0$ выполнялось условие

$$
u v+u^{*} v^{*}=\operatorname{sgn}\left(S_{x} S_{y}\right) .
$$


В результате для оператора энергии кислородных дырок получаем

$$
\begin{gathered}
H_{p}^{0}=\sum_{\vec{k}, \sigma}\left\{\epsilon_{a}(\vec{k}) a_{\vec{k}, \sigma}^{+} a_{\vec{k}, \sigma}+\epsilon_{b}(\vec{k}) b_{\vec{k}, \sigma}^{+} b_{\vec{k}, \sigma}\right\}, \\
\epsilon_{a, b}(\vec{k})=\epsilon_{p} \pm 4 t_{p p}\left|S_{x}(\vec{k}) S_{y}(\vec{k})\right| .
\end{gathered}
$$

Таким образом, гибридизация кислородных дырок привела к образованию двух подзон, одна из которых $\epsilon_{a}(\vec{k})$ лежит ниже, а вторая $\epsilon_{b}(\vec{k})$ - выше локального кислородного уровня $\epsilon_{p}$. Оставшийся произвол в выборе фазовых множителей коэффициентов $u(\vec{k}), v(\vec{k})$ может быть использован для установления наиболее удобной связи между кластерной орбиталью и диагонализующими фермионами. Сделаем следующий окончательный выбор коэффициентов $u(\vec{k}), v(\vec{k})$ :

$$
u(\vec{k})=\frac{i}{\sqrt{2}} \operatorname{sgn}\left(S_{x}(\vec{k})\right), \quad v(\vec{k})=-\frac{i}{\sqrt{2}} \operatorname{sgn}\left(S_{y}(\vec{k})\right) .
$$

Если мы введем ортогонализованные по узлам решетки Ванье амплитуды для диагонализующих фермионов

$$
\begin{aligned}
a_{i, \sigma} & =\frac{1}{\sqrt{N}} \sum_{\vec{k}} a_{\vec{k}, \sigma} e^{-i \vec{k} \vec{R}_{i}}, \\
b_{i, \sigma} & =\frac{1}{\sqrt{N}} \sum_{\vec{k}} b_{\vec{k}, \sigma} e^{-i \vec{k} \vec{R}_{i}},
\end{aligned}
$$

то сможем установить линейную связь между кластерной амплитудой $\beta_{i, \sigma}$ и диагонализуюшими амплитудами Ванье $a_{i, \sigma}, b_{i, \sigma}$ и соответствующие коэффициенты связи будут меньше единицы. Тогда для неортогонализованной кластерной амплитуды $\beta_{i, \sigma}$ получаem

$$
\begin{aligned}
\beta_{i, \sigma} & =\frac{1}{\sqrt{N}} \sum_{\vec{k}} e^{-i \vec{k} \vec{R}_{i}}\left[a_{\vec{k}, \sigma} \frac{\left|S_{x}\right|+\left|S_{y}\right|}{\sqrt{2}}+b_{\vec{k}, \sigma} \operatorname{sgn}\left(S_{x} S_{y}\right) \frac{\left|S_{y}\right|-\left|S_{x}\right|}{\sqrt{2}}\right]= \\
& =\sum_{j}\left[\lambda_{a}(i-j) a_{i, \sigma}+\lambda_{b}(i-j) b_{i, \sigma}\right]=\sum_{j} \lambda(i-j) P_{i, \sigma},
\end{aligned}
$$

где

$$
\begin{aligned}
\lambda_{a}(\vec{R}) & =\frac{1}{N} \sum_{\vec{k}} e^{-i \vec{k} \vec{R}} \frac{\left|S_{x}\right|+\left|S_{y}\right|}{\sqrt{2}}= \\
& =\frac{1}{2 \sqrt{2} \pi}\left[\frac{\delta_{y, 0}}{\frac{1}{4}-\left(\frac{x}{a}\right)^{2}}+\frac{\delta_{x, 0}}{\frac{1}{4}-\left(\frac{y}{a}\right)^{2}}\right] \\
\lambda_{b}(\vec{R}) & =\frac{1}{N} \sum_{\vec{k}} e^{-i \vec{k} \vec{R}} \operatorname{sgn}\left(S_{x} S_{y}\right) \frac{\left|S_{y}\right|-\left|S_{x}\right|}{\sqrt{2}}= \\
& =\frac{1}{\pi^{2}}\left[\frac{\left(1-\cos \pi \frac{x}{a}\right) \frac{y}{a} \cos \pi \frac{y}{a}}{\frac{x}{a}\left[\left(\frac{y}{a}\right)^{2}-\frac{1}{4}\right]}-\frac{\left(1-\cos \pi \frac{y}{a}\right) \frac{x}{a} \cos \pi \frac{x}{a}}{\frac{y}{a}\left[\left(\frac{x}{a}\right)^{2}-\frac{1}{4}\right]}\right] \\
\lambda(\vec{R}) & =\frac{1}{N} \sum_{\vec{k}} S(\vec{k}) e^{-i \vec{k} \vec{R}} .
\end{aligned}
$$


Здесь $\vec{R}=(x, y)$ - расстояние между узлами решетки меди и, следовательно, величины $x / a$ и $y / a$ равны целым положительным или отрицательным числам. Согласно (19) имеем $\lambda_{a}(0)=2 \sqrt{2} / \pi \simeq 0.900, \lambda_{b}(0)=0$.

\section{3. ВАНЬЕ-ПРЕДСТАВЛЕНИЕ ГАМИЛЬТОНИАНА СИСТЕМЫ}

На основании $(18)$ гамильтониан $(d-p)$-гибридизации приобретает вид

$$
\begin{aligned}
H_{d-p}= & 2 t_{d p} \lambda_{a}(0) \sum_{i}\left(d_{i \sigma}^{+} a_{i \sigma}+a_{i \sigma}^{+} d_{i \sigma}\right)+ \\
& +2 t_{d p} \sum_{j \neq i}\left(d_{i \sigma}^{+}\left[\lambda_{a}(i-j) a_{j \sigma}+\lambda_{b}(i-j) b_{j \sigma}\right]+\text { h.c. }\right) .
\end{aligned}
$$

Первое слагаемое в (20) соответствует главному локальному приближению, тогда как второе слагаемое, содержащее убьвающие с удалением от центрального иона меди функции $\lambda_{a}$ и $\lambda_{b}$, описывает процесс делокализации. В отличие от исходного гамильтониана этот член содержит вклады не только от ближайших соседей. Данное расширение пространств перескоков носит искусственный характер и соответствует процедуре узельной ортогонализации.

Из (19) $\left(\varepsilon_{1}, \varepsilon_{2}= \pm 1\right)$ имеем

$$
\begin{gathered}
\lambda_{a}\left(x=\varepsilon_{1} a, y=0\right)=\lambda_{a}\left(x=0, y=\varepsilon_{2} a\right)=-\frac{\lambda_{a}(0,0)}{6}, \\
\lambda_{a}\left(x=2 \varepsilon_{1} a, y=0\right)=\lambda_{a}\left(x=0, y=2 \varepsilon_{2} a\right)=-\frac{\lambda_{a}(0,0)}{30} \\
\lambda_{b}\left(x=\varepsilon_{1} a, y=0\right)=\lambda_{b}\left(x=0, y=\varepsilon_{2} a\right)=\lambda_{b}\left(x=\varepsilon_{1} a, y=\varepsilon_{2} a\right)=0 \\
\lambda_{b}\left(x=\varepsilon_{1} a, y=2 \varepsilon_{2} a\right)=-\lambda_{b}\left(x=2 \varepsilon_{1} a, y=\varepsilon_{1} a\right)=\frac{\varepsilon_{2}}{\varepsilon_{1}} \lambda_{a}(0,0) \frac{4 \sqrt{2}}{15 \pi} \\
\lambda_{b}\left(x=\varepsilon_{1} a, y=3 \varepsilon_{2} a\right)=-\lambda_{b}\left(x=3 \varepsilon_{1} a, y=\varepsilon_{2} a\right)=\frac{\varepsilon_{2}}{\varepsilon_{1}} \lambda_{a}(0,0) \frac{16 \sqrt{2}}{315 \pi}
\end{gathered}
$$

Функция $\lambda_{a}(x, y)$ отлична от нуля не только на центральном узле меди, но и на его ближайших соседях, быстро спадая на следующих соседних узлах. Функция $\lambda_{b}(x, y)$ равна нулю как на центральном, так и на ближайших соседних к нему узлах. Она отлична от нуля на следующих за ближайшими соседями узлах $x=\varepsilon_{1} a, y=2 \varepsilon_{2} a$ или $x=2 \varepsilon_{1} a$, $y=\varepsilon_{2} a\left(\right.$ где $\left.\varepsilon_{1,2}= \pm 1\right)$, затем она быстро спадает. Следовательно, сушествуют два канала делокализации дырок на меди, а именно, посредством их гибридизации как с $a_{i, \sigma^{-}}$, так и с $b_{i, \sigma}$-орбиталями.

Перейдем сейчас к гамильтониану $H_{p}^{0}$ свободных кислородных дырок и перепишем его в кластерном представлении,

$$
H_{p}^{0}=\sum_{i, j^{\prime}, \sigma}\left(t_{a}\left(j-j^{\prime}\right) a_{j, \sigma}^{+} a_{j^{\prime}, \sigma}+t_{b}\left(j-j^{\prime}\right) b_{j, \sigma}^{+} b_{j^{\prime}, \sigma}\right)
$$


где

$$
\begin{gathered}
t_{a, b}(\vec{R})=\frac{1}{N} \sum_{\vec{k}} e^{-i \vec{k} \vec{R}} \varepsilon_{a, b}(\vec{k})=\varepsilon_{p} \delta_{\vec{R}, 0} \pm 4 t_{p p} \tau(\vec{R}), \\
\tau(\vec{R})=\frac{1}{N} \sum_{\vec{k}} e^{-i \vec{k} \vec{R}}\left|S_{x} S_{y}\right|=\frac{1}{(2 \pi)^{2}\left[\frac{1}{4}-\left(\frac{x}{a}\right)^{2}\right]\left[\frac{1}{4}-\left(\frac{y}{a}\right)^{2}\right]} .
\end{gathered}
$$

На основании (22) получаем

$$
\tau(0)=\left(\frac{2}{\pi}\right)^{2}=0.4045, \quad \varepsilon_{a, b} \equiv \varepsilon_{p} \mp 4 t_{p p} \tau(0)
$$

и, следовательно, выражение (21) может быть переписано в виде

$$
H_{p}^{0}=\sum_{j, \sigma}\left(\varepsilon_{a} a_{j, \sigma}^{+} a_{j, \sigma}+\varepsilon_{b} b_{j, \sigma}^{+} b_{j, \sigma}\right)-4 t_{p p} \sum_{i \neq j^{\prime}, \sigma}\left(a_{j, \sigma}^{+} a_{j^{\prime}, \sigma}-b_{j, \sigma}^{+} b_{j^{\prime}, \sigma}\right) \tau\left(j-j^{\prime}\right) .
$$

Первое слагаемое в (23) представляет собой основной вклад от центрированных на узле меди амплитуд кислородных дырок, второе слагаемое описывает процесс их делокализации. Этот процесс протекает с одинаковой интенсивностью для обеих кислородных мод. Величина $\tau(\vec{R})$ быстро спадает с удалением от центрального узла решетки меди. Так, например,

$$
\tau( \pm a, 0)=\tau(0, \pm a)=-\frac{\tau(0,0)}{3}, \quad \tau( \pm a, \pm a)=\frac{\tau(0,0)}{9}
$$

Для перехода в остальных членах гамильтониана (1) к кластерному представлению выполним сначала этот переход в амплитудах кислородных дырок,

$$
\begin{aligned}
& p_{\vec{i} \pm \frac{a}{2} \hat{x}, \sigma}^{x}=\sum_{\vec{j}}\left[a_{\vec{j}, \sigma} \mu_{a}^{ \pm x}(\vec{i}-\vec{j})+b_{\vec{j}, \sigma} \mu_{b}^{ \pm x}(\vec{i}-\vec{j})\right], \\
& p_{\vec{i} \pm \frac{a}{2} \hat{y}, \sigma}^{y}=\sum_{\vec{j}}\left[a_{\vec{j}, \sigma} \mu_{a}^{ \pm y}(\vec{i}-\vec{j})+b_{\vec{j}, \sigma} \mu_{b}^{ \pm y}(\vec{i}-\vec{j})\right]
\end{aligned}
$$

где

$$
\begin{aligned}
& \mu_{a}^{ \pm x}(\vec{R})=\frac{1}{N} \sum_{\vec{k}} e^{-i \vec{k} \vec{R}} u(\vec{k}) e^{\mp i k_{x} \frac{a}{2}}=\frac{\delta_{y, 0}}{\sqrt{2} \pi\left(\frac{x}{a} \pm \frac{1}{2}\right)} \\
& \mu_{b}^{ \pm x}(\vec{R})=\frac{1}{N} \sum_{\vec{k}} e^{-i \vec{k} \vec{R}} v(\vec{k}) e^{\mp i k_{x} \frac{a}{2}}=\frac{\mp \cos \frac{\pi x}{a}\left(1-\cos \frac{\pi y}{a}\right)}{\sqrt{2} \pi^{2}\left(\frac{x}{a} \pm \frac{1}{2}\right)\left(\frac{y}{a}\right)} \\
& \mu_{a}^{ \pm y}(\vec{R})=-\frac{1}{N} \sum_{\vec{k}} e^{-i \vec{k} \vec{R}} v^{*}(\vec{k}) e^{\mp i k_{y} \frac{a}{2}}=-\frac{\delta_{x, 0}}{\sqrt{2} \pi\left(\frac{y}{a} \pm \frac{1}{2}\right)} \\
& \mu_{b}^{ \pm y}(\vec{R})=\frac{1}{N} \sum_{\vec{k}} e^{-i \vec{k} \vec{R}_{u^{*}}(\vec{k}) e^{\mp i k_{y} \frac{a}{2}}}=\frac{\mp \cos \frac{\pi y}{a}\left(1-\cos \frac{\pi x}{a}\right)}{\sqrt{2} \pi^{2}\left(\frac{y}{a} \pm \frac{1}{2}\right)\left(\frac{x}{a}\right)}
\end{aligned}
$$


Продолжая переход к кластерному представлению членов гамильтониана (1), рассмотрим кулоновское взаимодействие дырок на ионах кислорода. Соответствующий член гамильтониана имеет вид

$$
H_{p}^{c}=U_{p} \sum_{\vec{i}}\left[p_{x \uparrow}^{+} p_{x \uparrow} p_{x \downarrow}^{+} p_{x \downarrow}+p_{y \uparrow}^{+} p_{y \uparrow} p_{y \downarrow}^{+} p_{y \downarrow}\right],
$$

где для краткости использованы обозначения $p_{x}=p_{\vec{i}+\frac{a}{2} \hat{x}}, p_{y}=p_{\vec{i}+\frac{a}{2} \hat{y}}$. Подставим в правую часть (26) формулы (24) для операторов кислородных дырок:

$$
\begin{aligned}
H_{p}^{c}= & U_{p} \sum_{i} \sum_{j_{1}, j_{2}, j_{3}, j_{4}}\left\{\left[a_{j_{1} \uparrow}^{+}{ }^{*}{ }_{a}^{x}\left(i-j_{1}\right)+b_{j_{1} \uparrow}^{+}{ }^{*} \mu_{b}^{x}\left(i-j_{1}\right)\right] \times\right. \\
& \times\left[a_{j_{2} \uparrow} \mu_{a}^{x}\left(i-j_{2}\right)+b_{j_{2} \uparrow} \mu_{b}^{x}\left(i-j_{2}\right)\right]\left[a_{j_{3} \downarrow}^{+} \stackrel{*}{\mu}_{a}^{x}\left(i-j_{3}\right)+\right. \\
& \left.+b_{j_{3} \downarrow}^{+} \stackrel{*}{\mu}_{b}^{x}\left(i-j_{3}\right)\right]\left[a_{j_{4} \downarrow} \mu_{a}^{x}\left(i-j_{4}\right)+b_{j_{4} \downarrow} \mu_{b}^{x}\left(i-j_{4}\right)\right]+ \\
& +\left[a_{j_{1} \uparrow}^{+} \mu_{a}^{*}\left(i-j_{1}\right)+b_{j_{1} \uparrow}^{+}{ }^{*} \mu_{b}^{y}\left(i-j_{1}\right)\right]\left[a_{j_{2} \uparrow} \mu_{a}^{y}\left(i-j_{2}\right)+\right. \\
& \left.+b_{j_{2} \uparrow} \mu_{b}^{y}\left(i-j_{2}\right)\right]\left[a_{j_{3} \downarrow}^{+}{ }^{*} \mu_{a}^{y}\left(i-j_{3}\right)+b_{j_{3} \downarrow}^{+} \mu_{b}^{y}\left(i-j_{3}\right)\right] \times \\
& \left.\times\left[a_{j_{4} \downarrow} \mu_{a}^{y}\left(i-j_{4}\right)+b_{j_{4} \downarrow} \mu_{b}^{y}\left(i-j_{4}\right)\right]\right\} .
\end{aligned}
$$

Для выполнения суммирования по индексу узла меди воспользуемся фурье-представлениями (25) функций $\mu_{a(b)}^{x(y)}$. После этого преобразования возникает довольно громоздкое выражение, содержащее 16 членов, каждый из которых является произведением четырех фермионных операторов в ванье-представлении. Перечислим те из них, которые оказываются наиболее сушественными в локальном случае, т.е. при равенстве всех индексов узлов $j$,

$$
\begin{aligned}
H_{p}^{c}= & U_{p} \sum_{j_{1}, j_{2}, j_{3}, j_{4}}\left\{a_{j_{1} \uparrow}^{+} a_{j_{2} \uparrow} a_{j_{3} \downarrow}^{+} a_{j_{4} \downarrow} \Psi^{a a a a}\left(j_{1}-j_{2}, j_{3}-j_{4}, j_{2}-j_{4}\right)+\right. \\
& +b_{j_{1} \uparrow}^{+} b_{j_{2} \uparrow} b_{j_{3} \downarrow}^{+} b_{j_{4} \downarrow} \Psi^{b b b b}\left(j_{1}-j_{2}, j_{3}-j_{4}, j_{2}-j_{4}\right)+ \\
& +a_{j_{1} \uparrow}^{+} a_{j_{2} \uparrow} b_{j_{3} \downarrow}^{+} b_{j_{4} \downarrow} \Psi^{a a b b}\left(j_{1}-j_{2}, j_{3}-j_{4}, j_{2}-j_{4}\right)+ \\
& +b_{j_{1} \uparrow}^{+} b_{j_{2} \uparrow} a_{j_{3} \downarrow}^{+} a_{j_{4} \downarrow} \Psi^{b b a a}\left(j_{1}-j_{2}, j_{3}-j_{4}, j_{2}-j_{4}\right)+ \\
& +a_{j_{1} \uparrow}^{+} b_{j_{2} \uparrow} b_{j_{3} \downarrow}^{+} a_{j_{4} \downarrow} \Psi^{a b b a}\left(j_{1}-j_{4}, j_{3}-j_{2}, j_{4}-j_{2}\right)+ \\
& \left.+b_{j_{1} \uparrow}^{+} a_{j_{2} \uparrow} a_{j_{3} \downarrow}^{+} b_{j_{4} \downarrow} \Psi^{b a a b}\left(j_{1}-j_{4}, j_{3}-j_{2}, j_{4}-j_{2}\right)\right\},
\end{aligned}
$$


где

$$
\begin{aligned}
& \Psi^{a a a a}\left(j_{1}-j_{2}, j_{3}-j_{4}, j_{2}-j_{4}\right)=\frac{1}{N^{3}} \sum_{\vec{k}, \vec{k}^{\prime}, \vec{q}} e^{-i \vec{k}\left(\vec{j}_{1}-\vec{j}_{2}\right)} \times \\
& \times e^{-i \vec{k}^{\prime}\left(\vec{j}_{3}-\vec{j}_{4}\right)+i \vec{q}\left(\vec{j}_{2}-\vec{j}_{4}\right)}\left[u(\vec{k}) u(\vec{k}+\vec{q})^{*}\left(\vec{k}^{\prime}\right) u\left(\vec{k}^{\prime}-\vec{q}\right)+\right. \\
&\left.+v(\vec{k})^{*}(\vec{k}+\vec{q}) v\left(\vec{k}^{\prime}\right)^{*}\left(\vec{k}^{\prime}-\vec{q}\right)\right], \\
& \Psi^{a a b b}\left(j_{1}-j_{2}, j_{3}-j_{4}, j_{2}-j_{4}\right)=\frac{1}{N^{3}} \sum_{\vec{k}, \vec{k}^{\prime}, \vec{q}} e^{-i \vec{k}\left(\vec{j}_{1}-\vec{j}_{2}\right)} \times \\
& \quad \times e^{-i \vec{k}^{\prime}\left(\vec{j}_{3}-\vec{j}_{4}\right)+i \vec{q}\left(\vec{j}_{2}-\vec{j}_{4}\right)}\left[\stackrel{u}{u}(\vec{k}) u(\vec{k}+\vec{q})^{*}\left(\vec{k}^{\prime}\right) v\left(\vec{k}^{\prime}-\vec{q}\right)+\right. \\
&\left.+v(\vec{k})^{*}(\vec{k}+\vec{q}) u\left(\vec{k}^{\prime}\right) u\left(\vec{k}^{\prime}-\vec{q}\right)\right] .
\end{aligned}
$$

Остальные четыре коэффициента в (28) отличаются от приведенных в (29) заменой индексов $a$ и $b$ и функций $u(\vec{k})$ и $v(\vec{k})$ между собой. На основании определений $(16)$ следуют равенства

$$
\begin{aligned}
& \frac{1}{N^{3}} \sum_{\vec{k}, \vec{k}^{\prime}, \vec{q}} u(\vec{k}) u(\vec{k}+\vec{q})^{*}\left(\vec{k}^{\prime}\right) u\left(\vec{k}^{\prime}-\vec{q}\right)=\frac{1}{12}, \\
& \frac{1}{N^{3}} \sum_{\vec{k}, \vec{k}^{\prime}, \vec{q}} u(\vec{k}) u(\vec{k}+\vec{q}) v\left(\vec{k}^{\prime}\right) v\left(\vec{k}^{\prime}-\vec{q}\right)=\frac{1}{16},
\end{aligned}
$$

которые, будучи подставлены в (29), приводят к следующим значениям локальных коэффициентов:

$$
\begin{gathered}
\Psi^{a a a a}(0,0,0)=\Psi^{b b b b}(0,0,0)=\frac{1}{6} \\
\Psi^{a a b b}(0,0,0)=\Psi^{b b a a}(0,0,0)=\Psi^{a b b a}(0,0,0)=\Psi^{b a a b}(0,0,0)=\frac{1}{8} .
\end{gathered}
$$

Из приведенных вычислений следует, что основной вклад от кулоновского отталкивания кислородных дырок в ячейке, центрированной на узле меди, имеет вид

$$
\begin{aligned}
H_{p}^{c}= & U_{a} \sum_{j} n_{j \uparrow}^{a} n_{j \downarrow}^{a}+U_{b} \sum_{j} n_{j \uparrow}^{b} n_{j \downarrow}^{b}+ \\
& +U_{a b} \sum_{j}\left(n_{j \uparrow}^{a} n_{j \downarrow}^{b}+n_{j \uparrow}^{b} n_{j \downarrow}^{a}-S_{j+}^{a} S_{j-}^{b}-S_{j-}^{a} S_{j+}^{b}\right),
\end{aligned}
$$

где ренормированные константы $U_{a}=U_{b}=U_{p} / 6$ и $U_{a b}=U_{p} / 8$. В (32) используются определения операторов числа дырок $n_{j, \sigma}^{c}$ и переворота спина $S_{j \pm}^{c}$, где $c=a, b$,

$$
n_{j, \sigma}^{c}=c_{j, \sigma}^{+} c_{j, \sigma}, \quad S_{j+}^{c}=c_{j \uparrow}^{+} c_{j \downarrow}, \quad S_{j-}^{c}=c_{j \downarrow}^{+} c_{j \uparrow} .
$$

Последнее слагаемое в (32) может быть представлено в более простом виде

$$
U_{a b} \sum_{j}\left(a_{j \uparrow}^{+} b_{j \downarrow}^{+}-a_{j \downarrow}^{+} b_{j \uparrow}^{+}\right)\left(b_{j \downarrow} a_{j \uparrow}-b_{j \uparrow} a_{j \downarrow}\right),
$$


что демонстрирует взаимодействие $(a, b)$-синглетных пар. Гамильтониан $(26)$ и его ячеечное локальное представление (32) коммутируют с оператором спина $\vec{S}$ кислородных дырок и его квадратом как в исходном, так и в конечном представлении. В ячеечном локальном представлении оператор спина имеет вид

$$
\vec{S}^{a b}=\sum_{j}\left(\vec{S}_{j}^{a}+\vec{S}_{j}^{b}\right)
$$

где

$$
S_{j x}^{c}=\frac{1}{2}\left(S_{j+}^{c}+S_{j-}^{c}\right), \quad S_{j y}^{c}=\frac{1}{2 i}\left(S_{j+}^{c}-S_{j-}^{c}\right), \quad S_{j z}^{c}=\frac{1}{2}\left(n_{j \uparrow}^{c}-n_{j \downarrow}^{c}\right), \quad c=a, b
$$

Как видно из полученных значений энергий $U_{a}$ и $U_{a b}$, эффективное кулоновское отталкивание $a$ - и $b$-фермионов, центрированных на узле меди, сушественно меньше величины кулоновского отталкивания дырок, реально происходящего на ионах кислорода. Следует заметить, что уменьшение параметров $U_{a}$ и $U_{a b}$ по отношению к $U_{p}$, как и следовало ожидать, оказалось более значительным, чем соответствуюшее уменьшение при использовании канонических фермионов. В работе [22] приведено значение эффективного отталкивания в этом последнем случае: $\Psi(0,0,0) U_{p}=0.2109 U_{p}$. В предлагаемом выше подходе $U_{a}=0.1666 U_{p}$, а величина $U_{a b}=0.125 U_{p}$ оказалась еше меньше. Если воспользоваться стандартной системой значений параметров, типичных для купратов и приведенных в работах [19], [22],

$$
\begin{gathered}
t_{d p}=1.3{ }_{\ni} \mathrm{B}, \quad \frac{\varepsilon_{p}-\varepsilon_{d}}{t_{d p}}=2.7, \quad \frac{t_{p p}}{t_{d p}}=0.5, \\
\frac{U_{d}}{t_{d p}}=7, \quad \frac{U_{p}}{t_{d p}}=3, \quad \frac{U_{d p}}{t_{d p}}=1,
\end{gathered}
$$

то можно оценить полученные значения для эффективного отталкивания $U_{a}$ и $U_{a b}$ как величины, соразмерные с энергией гибридизации дырок кислорода $U_{a}=t_{p p}$ и $U_{a b}=$ $\frac{3}{4} t_{p p}$. Следовательно, оба эти эффекта должны рассматриваться одновременно. Поэтому представляется неправомерным учет кулоновского отталкивания $U_{p}$ дырок на кислороде и одновременное пренебрежение матричными элементами $t_{p p}$ межкислородного туннелирования. Наряду с эффективным ячеечным гамильтонианом (32) оператор (28) содержит также межъячеечные слагаемые, которые описывают процессы делокализации дырок, обусловленные кулоновским отталкиванием $H_{p}^{c}$ дырок на кислороде. Все эти слагаемые имеют несовпадаюшие индексы узлов решетки меди. Следует ожидать, что нелокальные вклады в (28) в несколько раз меньше, чем локальный вклад (32), который сам по себе оказался сильно ослабленным по сравнению с реальным отталкиванием $U_{p}$. Эти нелокальные слагаемые будут в дальнейшем опушены. Одновременно будут опушены нелокальные вклады, связанные с межкислородной гибридизацией дырок. 
Рассмотрим, наконец, преобразование слагаемого исходного гамильтониана (1), которое описывает кулоновское отталкивание дырок на соседних ионах меди и кислорода. Перепишем это слагаемое в виде

$$
H_{d-p}^{c}=U_{d p} \sum_{i, \sigma, \sigma^{\prime}} d_{i, \sigma}^{+} d_{i, \sigma}\left(p_{x, \sigma^{\prime}}^{+} p_{x, \sigma^{\prime}}+p_{-x, \sigma^{\prime}}^{+} p_{-x, \sigma^{\prime}}+p_{y, \sigma^{\prime}}^{+} p_{y, \sigma^{\prime}}+p_{-y, \sigma^{\prime}}^{+} p_{-y, \sigma^{\prime}}\right) \text {. }
$$

Здесь используются краткие обозначения (26) для операторов $p_{x, \sigma}$ и $p_{y, \sigma}$. Подставив в (38) формулы (24) и выполнив несложные преобразования, получим выражение, содержашее операторы орбиталей, центрированных на узлах решетки меди,

$$
\begin{aligned}
H_{d p}^{c}= & U_{d p} \sum_{i, j, j^{\prime}} \sum_{\sigma, \sigma^{\prime}} n_{i \sigma}^{d}\left[\phi^{a a}\left(i-j ; i-j^{\prime}\right) a_{j \sigma^{\prime}}^{+} a_{j^{\prime} \sigma^{\prime}}+\phi^{a b}\left(i-j ; i-j^{\prime}\right) a_{j \sigma^{\prime}}^{+} b_{j^{\prime} \sigma^{\prime}}+\right. \\
& \left.+\phi^{b a}\left(i-j ; i-j^{\prime}\right) b_{j \sigma^{\prime}}^{+} a_{j^{\prime} \sigma^{\prime}}+\phi^{b b}\left(i-j ; i-j^{\prime}\right) b_{j \sigma^{\prime}}^{+} b_{j^{\prime} \sigma^{\prime}}\right] .
\end{aligned}
$$

Коэффициенты этого выражения определены следующим образом:

$$
\begin{aligned}
\phi^{a a}(i- & \left.j ; i-j^{\prime}\right)=\frac{1}{N^{2}} \sum_{\vec{k}, \vec{k}^{\prime}} e^{i \vec{k}\left(\vec{R}_{i}-\vec{R}_{j}\right)-i \vec{k}^{\prime}\left(\vec{R}_{i}-\vec{R}_{j^{\prime}}\right)} \times \\
\times & {\left[2 \cos \frac{\left(k_{x}-k_{x}^{\prime}\right) a}{2} u(\vec{k}) u\left(\vec{k}^{\prime}\right)+2 \cos \frac{\left(k_{y}-k_{y}^{\prime}\right) a}{2} v(\vec{k})^{*} v\left(\vec{k}^{\prime}\right)\right], } \\
\phi^{a b}(i- & \left.j ; i-j^{\prime}\right)=\frac{1}{N^{2}} \sum_{\vec{k}, \vec{k}^{\prime}} e^{i \vec{k}\left(\vec{R}_{i}-\vec{R}_{j}\right)-i \vec{k}^{\prime}\left(\vec{R}_{i}-\vec{R}_{j^{\prime}}\right)} \times \\
\times & {\left[2 \cos \frac{\left(k_{x}-k_{x}^{\prime}\right) a}{2} u(\vec{k}) v\left(\vec{k}^{\prime}\right)-2 \cos \frac{\left(k_{y}-k_{y}^{\prime}\right) a}{2} v(\vec{k}) u^{*}\left(\vec{k}^{\prime}\right)\right], } \\
\phi^{b b}(i- & \left.j i i-j^{\prime}\right)=\frac{1}{N^{2}} \sum_{\vec{k}, \vec{k}^{\prime}} e^{i \vec{k}\left(\vec{R}_{i}-\vec{R}_{j}\right)-i \vec{k}^{\prime}\left(\vec{R}_{i}-\vec{R}_{j^{\prime}}\right) \times} \\
\times & {\left[2 \cos \frac{\left(k_{x}-k_{x}^{\prime}\right) a}{2} v(\vec{k}) v\left(\vec{k}^{\prime}\right)+2 \cos \frac{\left(k_{y}-k_{y}^{\prime}\right) a}{2} u(\vec{k}) u^{*}\left(\vec{k}^{\prime}\right)\right], } \\
\phi^{b a}(i- & \left.j ; i-j^{\prime}\right)=\left(\phi^{a b}\left(i-j^{\prime} ; i-j\right)\right)^{*} .
\end{aligned}
$$

В локальном случае, т.е. при $i=j=j^{\prime}$, из четырех коэффициентов (40) только первый оказывается отличным от нуля,

$$
\phi^{a a}=\phi^{a a}(0,0)=\frac{8}{\pi^{2}} \simeq 0.811 .
$$

Следовательно, в ячейке, центрированной на узле $i$, проявляется кулоновское отталкивание только между диагонализующими $a_{i}$-фермионами и $d_{i}$-дырками. Вклад от диагонализующих $b_{i}$-фермионов при этом отсутствует, как это видно из выражения

$$
\begin{gathered}
H_{a d}^{c}=U_{a d} \sum_{i} n_{i}^{d} n_{i}^{a}, \\
U_{a d}=U_{d p} \phi^{a a}=\frac{8 U_{d p}}{\pi^{2}}, \quad n_{i}^{\alpha}=\sum_{\sigma} n_{i \sigma}^{\alpha}, \quad \alpha=a, d .
\end{gathered}
$$


Соответствующее выражение, полученное в работе [22] при использовании канонических фермионов, привело к несколько большему значению эффективной константы отталкивания, а именно $\varphi_{000} U_{d p}=0.9180 U_{d p}$.

Приведем здесь результаты вычислений коэффициентов (40), которые будут использоваться в дальнейшем. На основании значений (16) для величин $u(\vec{k})$ и $v(\vec{k})$ нетрудно получить, что

$$
\begin{aligned}
& \varphi^{a a}\left(\vec{R}, \vec{R}^{\prime}\right)= \frac{1}{\pi^{2}}\left\{\frac{\delta_{x, 0} \delta_{x^{\prime}, 0}\left(\frac{y}{a} \frac{y^{\prime}}{a}+\frac{1}{4}\right)}{\left[\left(\frac{y}{a}\right)^{2}-\frac{1}{4}\right]\left[\left(\frac{y^{\prime}}{a}\right)^{2}-\frac{1}{4}\right]}+\left(\begin{array}{c}
x \rightarrow y \\
x^{\prime} \rightarrow y^{\prime}
\end{array}\right)\right\}, \\
& \varphi^{a b}\left(\vec{R}, \vec{R}^{\prime}\right)=-\frac{1}{2 \pi^{3}}\left\{\frac{\delta_{x, 0}\left[1-\cos \left(\frac{\pi x^{\prime}}{a}\right)\right] \cos \left(\frac{\pi y^{\prime}}{a}\right)\left(\frac{y}{a}+\frac{y^{\prime}}{a}\right)}{\left(\frac{x^{\prime}}{a}\right)\left[\left(\frac{y}{a}\right)^{2}-\frac{1}{4}\right]\left[\left(\frac{y^{\prime}}{a}\right)^{2}-\frac{1}{4}\right]}-\left(\begin{array}{c}
x \rightarrow y \\
x^{\prime} \rightarrow y^{\prime}
\end{array}\right)\right\}, \\
& \varphi^{b b}\left(\vec{R}, \vec{R}^{\prime}\right)=\frac{1}{\pi^{4}}\left\{\frac{\left[1-\cos \left(\frac{\pi x^{\prime}}{a}\right)\right]\left[1-\cos \left(\frac{\pi x}{a}\right)\right] \cos \left(\frac{\pi y}{a}\right) \cos \left(\frac{\pi y^{\prime}}{a}\right)}{\left(\frac{x}{a}\right)\left(\frac{x^{\prime}}{a}\right)\left[\left(\frac{y}{a}\right)^{2}-\frac{1}{4}\right]\left[\left(\frac{y^{\prime}}{a}\right)^{2}-\frac{1}{4}\right]} \times\right. \\
&\left.\times\left(\frac{y}{a} \frac{y^{\prime}}{a}+\frac{1}{4}\right)-\left(\begin{array}{c}
x \rightarrow y \\
x^{\prime} \rightarrow y^{\prime}
\end{array}\right)\right\} .
\end{aligned}
$$

Здесь $x\left(x^{\prime}\right)$ и $y\left(y^{\prime}\right)$ - проекции вектора $\vec{R}\left(\vec{R}^{\prime}\right)$. При этом считается, что отношения $x / a\left(x^{\prime} / a\right), y / a\left(y^{\prime} / a\right)$ - целые положительные или отрицательные числа. Как видно из полученных выражений, межузельная $(d-p)$-кулоновская энергия отталкивания трансформируется к ячеечному представлению без сушественного своего ослабления. После выделения локальной части (42) оператор (39) может быть представлен в виде суммы этого основного слагаемого и величины, определяюшей межузельные перескоки дырок,

$$
H_{d p}^{c}=H_{a d}^{c}+H_{d p}^{\prime}
$$

где $H_{d p}^{\prime}$ получается из гамильтониана (39), если в последнем опустить слагаемые с совпадаюшими индексами.

Данный этап вычислений завершает преобразование к ячеечному представлению тех слагаемых гамильтониана (1), которые содержат кислородные дырки, образуюшие кластер вокруг иона меди. После такого преобразования определяются как главные вклады, локализованные на ячейках, центрированных на узлах меди, так и межъячеечные слагаемые, содержашие, вообше говоря, дальнодействуюшее туннелирование ортогонализованных ванье-фермионов. В результате исходный гамильтониан (1) может быть представлен в виде суммы основного локального вклада $H^{0}$ и слагаемого $H^{\prime}$, определяюшего процесс делокализации как $d$-, так и диагонализуюших $a$ - и $b$-дырок,

$$
H=H^{0}+H^{\prime}, \quad H^{0}=\sum_{i} H_{i}^{0}
$$


где одноячеечньй локальный вклад

$$
\begin{aligned}
H^{0}= & \epsilon_{d} \sum_{\sigma} n_{\sigma}^{d}+U_{d} n_{\uparrow}^{d} n_{\downarrow}^{d}+\epsilon_{a} \sum_{\sigma} n_{\sigma}^{a}+U_{a} n_{\uparrow}^{a} n_{\downarrow}^{a}+\epsilon_{b} \sum_{\sigma} n_{\sigma}^{b}+U_{b} n_{\uparrow}^{b} n_{\downarrow}^{b}+ \\
& +t_{a d} \sum_{\sigma}\left(d_{\sigma}^{+} a_{\sigma}+a_{\sigma}^{+} d_{\sigma}\right)+U_{a d} n^{d} n^{a}+U_{a b}\left(n_{\uparrow}^{a} n_{\downarrow}^{b}+n_{\downarrow}^{a} n_{\uparrow}^{a}-S_{+}^{a} S_{-}^{b}-S_{-}^{a} S_{+}^{b}\right) .
\end{aligned}
$$

Здесь для простоты опушен индекс $i$ узла меди и подразумеваются равенства $U_{b}=U_{a}$, $U_{a b}=\frac{3}{4} U_{a}, \epsilon_{a, b}=\epsilon_{p} \mp 4 t_{p p} \tau(0)$. Возмущающий гамильтониан $H^{\prime}$ имеет довольно сложньй вид, так как он содержит многочисленные слагаемые, которые описывают квантовые переходы $d-, a$ - и $b$-фермионов между различными ячейками. Мы выделим в качестве наиболее сушественных следуюшие два слагаемых и опустим остальные:

$$
\begin{aligned}
H^{\prime}= & 2 t_{d p} \sum_{j \neq i}\left[d_{i \sigma}^{+}\left(\lambda_{a}(i-j) a_{j \sigma}+\lambda_{b}(i-j) b_{j \sigma}\right)+\text { h.c. }\right]+ \\
& +U_{d p} \sum_{i, j, j^{\prime}}^{\prime} \sum_{\sigma} n_{i}^{d}\left[\phi^{a a}\left(i-j, i-j^{\prime}\right) a_{j \sigma}^{+} a_{j^{\prime} \sigma}+\phi^{a b}\left(i-j, i-j^{\prime}\right) a_{j \sigma}^{+} b_{j^{\prime} \sigma}+\right. \\
& \left.+\phi^{b a}\left(i-j, i-j^{\prime}\right) b_{j \sigma}^{+} a_{j^{\prime} \sigma}+\phi^{b b}\left(i-j, i-j^{\prime}\right) b_{j \sigma}^{+} b_{j^{\prime} \sigma}\right]
\end{aligned}
$$

Здесь штрих у суммы подразумевает отсутствие слагаемых с тремя совпадаюшими индексами $\left(i=j=j^{\prime}\right)$ узлов решетки меди. В выражение (47) не включены слагаемые, обязанные матричным элементам перескоков $\tau_{p p}$ и эффективному кулоновскому отталкиванию $U_{a}$ и $U_{a b}$ ввиду их меньшей значимости.

\section{4. ДИАГОНАЛИЗАЦИЯ ЯЧЕЕЧНОГО ГАМИЛЬТОНИАНА И ОБСУЖДЕНИЕ РЕЗУЛЬТАТОВ}

Как видно из выражения (46), в каждой ячейке, центрированной на узлах меди, имеются три вида сильно коррелированных фермионов, а именно $d$-дырки на ионах меди и диагонализующие фермионы $a$ и $b$, описьвающие движение кислородных дырок в $\mathrm{CuO}_{2}$-плоскости. Каждый из трех видов фермионов, если считать их независимыми, обладает четырьмя квантовыми состояниями $|0\rangle,|\uparrow\rangle,|\downarrow\rangle,|\uparrow \downarrow\rangle$ с энергиями $E_{0}^{\alpha}=0$, $E_{\sigma}^{\alpha}=\varepsilon_{\alpha}, E_{2}^{\alpha}=2 \varepsilon_{\alpha}+U_{\alpha}$, где $\alpha=a, b, d$. Полное число квантовых состояний ячейки, равное числу состояний независимых $a$-, $b$ - и $d$-фермионов, есть $4^{3}=64$. Поэтому матрица гамильтониана (46), построенная на состояниях $\phi_{A}$ этих частиц, представляется довольно громоздким образованием. Индекс $A$ квантового состояния содержит числа частиц $N_{a}, N_{b}$ и $N_{d}$ системы, их полный спин $S$ и проекцию спина $S_{z}$. На самом деле целесообразно использовать вместо трех чисел $N_{\alpha}$ суммарное значение числа частиц состояния $N=N_{a}+N_{b}+N_{d}$ и два других независимых числа, скажем, $N_{a}$ и $N_{b}$. Это связано с тем, что ренормированные состояния гамильтониана (46), обозначенные нами через $\left|E_{\lambda}\right\rangle$, являются линейными комбинациями функций $\phi_{A}$, которые сохраняют числа 
$N, S$ и $S_{z}$, но не сохраняют отдельные значения числа частиц. Таким образом, набор квантовых чисел $\lambda$, помимо упомянутых, содержит также и дополнительные числа. На самом деле рассматриваемая матрица является квазидиагональной, и проблема определения ренормированных квантовых состояний и их энергий сводится к диагонализации субматриц с небольшим числом строк и столбцов (не большим четырех).

Для непосредственного определения ренормированных величин воспользуемся методом канонического преобразования, предложенным в работах [44]-[46] по периодической модели Андерсона. Метод основан на введении ортонормированной системы функций $\Phi_{n}\left(E_{\lambda}\right)$, удовлетворяюшей матричному уравнению

$$
\left(\widehat{H}^{0}-E_{\lambda} \hat{I}\right)\left(\begin{array}{c}
\Phi_{1}\left(E_{\lambda}\right) \\
\vdots \\
\Phi_{n}\left(E_{\lambda}\right)
\end{array}\right)=0
$$

и условиям ортонормировки

$$
\begin{aligned}
& \sum_{\lambda} \Phi_{n}^{*}\left(E_{\lambda}\right) \Phi_{m}\left(E_{\lambda}\right)=\delta_{n m}, \\
& \sum_{n} \Phi_{n}^{*}\left(E_{\lambda}\right) \Phi_{n}\left(E_{\lambda^{\prime}}\right)=\delta_{\lambda \lambda^{\prime}} .
\end{aligned}
$$

Здесь $\widehat{H}^{0}$ - матрица гамильтониана, построенная на функциях независимых частиц, $\hat{I}-$ единичный оператор.

Связь между исходными $\Psi_{n}$ и ренормированными $\left|E_{\lambda}\right\rangle$ состояниями имеет вид

$$
\Psi_{n}=\sum_{\lambda} \Phi_{n}\left(E_{\lambda}\right)\left|E_{\lambda}\right\rangle, \quad\left|E_{\lambda}\right\rangle=\sum_{n} \Phi_{n}^{*}\left(E_{\lambda}\right) \Psi_{n}
$$

Перейдем к детальному анализу ренормированных состояний в соответствии с принятой классификацией. Состояние с $N=0$ соответствует вакуумному состоянию. Оно обозначается через $|0\rangle$ и не ренормируется взаимодействиями, содержашимися в $H^{0}$. Энергия этого состояния равна нулю.

При $N=1$ имеются три функции независимых частиц со спином $S=1 / 2$ и его проекцией $S_{z}=1 / 2$ вида

$$
\Psi_{1}=d_{\uparrow}^{+}|0\rangle, \quad \Psi_{2}=a_{\uparrow}^{+}|0\rangle, \quad \Psi_{3}=b_{\uparrow}^{+}|0\rangle
$$

Нетрудно видеть, что действие гамильтониана $H^{0}$ на эти функции приводит к следующим равенствам:

$$
H^{0} \Psi_{1}=\varepsilon_{d} \Psi_{1}+V \Psi_{2}, \quad H^{0} \Psi_{2}=\varepsilon_{a} \Psi_{2}+V \Psi_{1}, \quad H^{0} \Psi_{3}=\varepsilon_{b} \Psi_{3}
$$


Для краткости по аналогии с работами [44], [45] введено обозначение $V=t_{a d}$. Соответствуюшая субматрица второго ранга в подпространстве состояний $\Psi_{1}$ и $\Psi_{2}$ имеет вид

$$
\left(\begin{array}{cc}
\varepsilon_{d}-E & V \\
V & \varepsilon_{a}-E
\end{array}\right)
$$

(здесь и в дальнейшем приводится подматрица матрицы $\widehat{H}^{0}-E \hat{I}$ ). На ее основе, полагая соответствующий детерминант равным нулю, получаем два значения энергии из ренормированного энергетического спектра дублета,

$$
E_{1,2}\left(N=1, S=\frac{1}{2}\right)=E_{1,2}=\frac{1}{2}\left[\varepsilon_{a}+\varepsilon_{d} \mp \sqrt{\left(\varepsilon_{a}-\varepsilon_{d}\right)^{2}+4 V^{2}}\right] .
$$

На основе (53) определим две ортонормированные функции $\Phi_{1}\left(E_{\lambda}\right)$ и $\Phi_{2}\left(E_{\lambda}\right)$ по правилам (48). Нетрудно видеть, что

$$
\begin{aligned}
\Phi_{2}\left(E_{\lambda}\right) & =\frac{E_{\lambda}-\varepsilon_{d}}{V} \Phi_{1}\left(E_{\lambda}\right), \\
\Phi_{1}\left(E_{1,2}\right) & =\frac{1}{\sqrt{2}}\left[1 \mp \frac{\varepsilon_{a}-\varepsilon_{d}}{\sqrt{\left(\varepsilon_{a}-\varepsilon_{d}\right)^{2}+4 V^{2}}}\right]^{\frac{1}{2}}
\end{aligned}
$$

и, следовательно, ренормированные состояния дублета суть

$$
\begin{aligned}
& \left|E_{1}\right\rangle=\Phi_{1}\left(E_{1}\right)\left[\Psi_{1}+\frac{E_{1}-\varepsilon_{d}}{V} \Psi_{2}\right] \\
& \left|E_{2}\right\rangle=\Phi_{1}\left(E_{2}\right)\left[\Psi_{1}+\frac{E_{2}-\varepsilon_{d}}{V} \Psi_{2}\right] .
\end{aligned}
$$

В этих формулах, как и далее, мы для краткости опускаем значения квантовых чисел $N, S$ и $S_{z}$ и учитываем вешественность функции $\Phi_{n}$. Функция $\Psi_{3}$ является собственной функцией оператора $H^{0}, H^{0} \Psi_{3}=\epsilon_{b} \Psi_{3}$ и, следовательно, $E_{3}=\epsilon_{b},\left|E_{3}\right\rangle=\Psi_{3}$. Имеются еше три функции $\Psi_{4}, \Psi_{5}$ и $\Psi_{6}$, которые отличаются от рассмотренных направлением спина частиц. Соответствуюшие им ренормированные величины имеют тот же вид, что и для функций $\Psi_{1}, \Psi_{2}$ и $\Psi_{3}$, соответственно. Поэтому можно написать, что $E_{4,5}=E_{1,2}$, $E_{6}=E_{3}, \Phi_{4,5}=\Phi_{1,2}, \Phi_{6}=1$.

Перейдем теперь к состояниям с двумя частицами, $N=2$. Имеются два возможных значения полного спина $S=0$ и $S=1$. Здесь мы рассмотрим первый случай. При $S=0$ имеются шесть синглетных состояний $\Psi(N=2, S=0, n)$ с $n=1, \ldots, 6$, которые перечислены ниже (для простоты опускаем индексы $N$ и $S$ ):

$$
\begin{aligned}
\Psi_{1} & =\frac{1}{\sqrt{2}}\left(d_{\uparrow}^{+} a_{\downarrow}^{+}-d_{\downarrow}^{+} a_{\uparrow}^{+}\right)|0\rangle, & \Psi_{2} & =a_{\uparrow}^{+} a_{\downarrow}^{+}|0\rangle, \\
\Psi_{3} & =d_{\uparrow}^{+} d_{\downarrow}^{+}|0\rangle, & \Psi_{4} & =\frac{1}{\sqrt{2}}\left(d_{\uparrow}^{+} b_{\downarrow}^{+}-d_{\downarrow}^{+} b_{\uparrow}^{+}\right)|0\rangle, \\
\Psi_{5} & =\frac{1}{\sqrt{2}}\left(a_{\uparrow}^{+} b_{\downarrow}^{+}-a_{\downarrow}^{+} b_{\uparrow}^{+}\right)|0\rangle, & \Psi_{6} & =b_{\uparrow}^{+} b_{\downarrow}^{+}|0\rangle .
\end{aligned}
$$


Действие гамильтониана (46) на эти функции приводит к следующим равенствам:

$$
\begin{gathered}
H^{0} \Psi_{1}=\left(\varepsilon_{a}+\varepsilon_{d}+G\right) \Psi_{1}+\sqrt{2} V \Psi_{2}+\sqrt{2} V \Psi_{3}, \\
H^{0} \Psi_{2}=\left(2 \varepsilon_{a}+U_{a}\right) \Psi_{2}+\sqrt{2} V \Psi_{1}, \\
H^{0} \Psi_{3}=\left(2 \varepsilon_{d}+U_{d}\right) \Psi_{3}+\sqrt{2} V \Psi_{1}, \quad H^{0} \Psi_{4}=\left(\varepsilon_{d}+\varepsilon_{b}\right) \Psi_{4}+V \Psi_{5}, \\
H^{0} \Psi_{5}=\left(\varepsilon_{a}+\varepsilon_{b}+2 U_{a b}\right) \Psi_{5}+V \Psi_{4}, \quad H^{0} \Psi_{6}=\left(2 \varepsilon_{b}+U_{b}\right) \Psi_{6} .
\end{gathered}
$$

Согласно этим равенствам матрица гамильтониана (46) в подпространстве первых трех состояний имеет вид

$$
\left(\begin{array}{ccc}
\varepsilon_{a}+\varepsilon_{d}+G-E & \sqrt{2} V & \sqrt{2} V \\
\sqrt{2} V & 2 \varepsilon_{a}+U_{a}-E & 0 \\
\sqrt{2} V & 0 & 2 \varepsilon_{d}+U_{d}-E
\end{array}\right)
$$

где использовано обозначение работ [44], [45] $G=U_{a d}$. Три значения энергии синглетов, образованных $a$ - и $d$-фермионами, находятся из решения кубического уравнения относительно $E$,

$$
\begin{gathered}
\left(2 \varepsilon_{a}+U_{a}-E\right)\left(2 \varepsilon_{d}+U_{d}-E\right)\left(\varepsilon_{a}+\varepsilon_{d}+G-E\right)- \\
-2 V^{2}\left(2 \varepsilon_{a}+U_{a}+2 \varepsilon_{d}+U_{d}-2 E\right)=0
\end{gathered}
$$

(синглет Жанга-Райса [4] имеет наименьшую энергию). Функции $\Phi_{n}\left(E_{\lambda}\right)$ в данном случае имеют вид

$$
\begin{aligned}
& \Phi_{1}\left(E_{\lambda}\right)=\left(1+\frac{2 V^{2}}{\left(2 \varepsilon_{a}+U_{a}-E_{\lambda}\right)^{2}}+\frac{2 V^{2}}{\left(2 \varepsilon_{d}+U_{d}-E_{\lambda}\right)^{2}}\right)^{\frac{1}{2}}, \\
& \Phi_{2}\left(E_{\lambda}\right)=-\frac{\sqrt{2} V \Phi_{1}\left(E_{\lambda}\right)}{2 \varepsilon_{a}+U_{a}-E_{\lambda}}, \quad \Phi_{3}\left(E_{\lambda}\right)=-\frac{\sqrt{2} V \Phi_{1}\left(E_{\lambda}\right)}{2 \varepsilon_{d}+U_{d}-E_{\lambda}},
\end{aligned}
$$

и, следовательно, три ренормированных состояния $(\lambda=1,2,3)$

$$
\left|E_{\lambda}\right\rangle=\frac{\Phi_{1}\left(E_{\lambda}\right)}{\sqrt{2}}\left[d_{\uparrow}^{+} a_{\downarrow}^{+}-d_{\downarrow}^{+} a_{\uparrow}^{+}-\frac{2 V a_{\uparrow}^{+} a_{\downarrow}^{+}}{2 \varepsilon_{a}+U_{a}-E_{\lambda}}-\frac{2 V d_{\uparrow}^{+} d_{\downarrow}^{+}}{2 \varepsilon_{d}+U_{d}-E_{\lambda}}\right]|0\rangle .
$$

Для подсистемы функций $\Psi_{4}$ и $\Psi_{5}$ матрица ячеечного гамильтониана есть

$$
\left(\begin{array}{cc}
\varepsilon_{d}+\varepsilon_{b}-E & V \\
V & \varepsilon_{a}+\varepsilon_{b}+2 U_{a b}-E
\end{array}\right) .
$$

Отсюда следуют два значения энергии

$$
E_{4,5}(2,0)=\varepsilon_{b}+\frac{1}{2}\left[\varepsilon_{a}+\varepsilon_{d}+2 U_{a b} \mp \sqrt{\left(\varepsilon_{d}-\varepsilon_{a}-2 U_{a b}\right)^{2}+4 V^{2}}\right]
$$


$(d, b)$ - и $(a, b)$-синглетов, построенных в виде $(a, d)$-дублета $(54)$ и одного $b$-фермиона с заменой энергии $\varepsilon_{a}$ на $\varepsilon_{a}+2 U_{a b}$. Здесь могут быть использованы выражения $(55)$ с необходимой модификацией для получения интересуюших нас величин $\Phi_{n}\left(E_{\lambda}\right)$,

$$
\begin{aligned}
\Phi_{5}\left(E_{\lambda}\right) & =\frac{\left(E_{\lambda}-\varepsilon_{d}-\varepsilon_{b}\right)}{V} \Phi_{4}\left(E_{\lambda}\right) \\
\Phi_{4}\left(E_{4,5}\right) & =\frac{1}{2}\left[1 \pm \frac{\varepsilon_{a}+2 U_{a b}-\varepsilon_{d}}{\sqrt{\left(\varepsilon_{a}+2 U_{a b}-\varepsilon_{d}\right)^{2}+4 V^{2}}}\right]^{\frac{1}{2}}
\end{aligned}
$$

На этом основании получаем

$$
\left|E_{4,5}\right\rangle=\Phi_{4}\left(E_{4,5}\right)\left(\Psi_{4}+\frac{E_{4,5}-\varepsilon_{d}-\varepsilon_{b}}{V} \Psi_{5}\right)
$$

Синглет $\Psi_{6}$ не ренормируется и состоит из двух $b$-частищ с противоположными спинами. Энергия этого состояния $E_{6}=2 \varepsilon_{b}+U_{b}$, и выполняется равенство $\left|E_{6}\right\rangle=\Psi_{6}$.

Если воспользоваться стандартной системой значений параметров теории (37), то уровни энергии состояний с $N=1$ становятся

$$
\begin{aligned}
& E_{1}=\varepsilon_{d}-1.088 t_{d p}=\varepsilon_{d}-1.414{ }^{\ni B}, \\
& E_{2}=\varepsilon_{d}+2.978 t_{d p}=\varepsilon_{d}+3.871{ } \mathrm{~B}, \\
& E_{3}=\varepsilon_{d}+3.510 t_{d p}=\varepsilon_{d}+4.563{ } \mathrm{~B} .
\end{aligned}
$$

В отсутствие диагонализации эти же уровни энергии имеют несколько иные значения:

$$
\begin{aligned}
& \bar{E}_{1}=\varepsilon_{d}-0.994 t_{d p}=\varepsilon_{d}-1.292 \text { эВ, } \\
& \bar{E}_{2}=\varepsilon_{d}+3.694 t_{d p}=\varepsilon_{d}+4.802 \text { эB, } \\
& \bar{E}_{3}=\varepsilon_{d}+2.700 t_{d p}=\varepsilon_{d}+3.510 \ni \mathrm{B} .
\end{aligned}
$$

Нижний уровень дублета из-за введения диагонализации оказывается несколько ниже (на 0.122 эВ), чем при ее отсутствии. Аналогичный результат получен для синглета Жанга-Райса. В самом деле, уравнение (60), определяющее три уровня синглетов, для значений параметров (37) переписывается в виде

$$
\begin{gathered}
E_{n}=2 \varepsilon_{d}+e_{n} t_{d p}, \quad n=1,2,3 \\
(7-e)(2.701-e)(4.280-e)-6.48(11.280-2 e)=0 .
\end{gathered}
$$

Это кубическое уравнение имеет решения

$$
e_{1}=0.174, \quad e_{2}=5.294, \quad e_{3}=8.513
$$


В отсутствие перенормировки кислородных дырок кубическое уравнение и его решения оказываются иными,

$$
\begin{gathered}
(7-\bar{e})(3.618-\bar{e})(6.033-\bar{e})-7.342(13.033-2 \bar{e})=0, \\
\bar{e}_{1}=0.957, \quad \bar{e}_{2}=6.471, \quad \bar{e}_{3}=9.223 .
\end{gathered}
$$

Из сопоставления этих решений видно, что различие между энергиями синглета Жанга-Райса в этих двух случаях $E_{1}-\bar{E}_{1}=-1.018$ эВ оказалось существенным.

Таким образом, можно сделать вывод, что выполненные нами диагонализация кинетической энергии кислородных дырок и их перенормировка способствуют понижению энергии низкоэнергетической части спектра и должны оказать существенное влияние на динамические свойства системы.

Благодарности. Авторы выражают глубокую признательность проф. Н. М. Плакиде за неоднократные обсуждения различных аспектов работы. Мы благодарим Высший Совет по науке и технологическому развитию Республики Молдова за предоставленные гранты. Один из авторов (В.М.) благодарит за поддержку Программу Гейзенберг-Ландау.

\section{Список литературы}

[1] V. J. Emery. Phys. Rev. Lett. 1987. V. 58. P. 2794.

[2] C. M. Varma, S. Schmitt-Rink, E. Abrahams. Solid State Commun. 1987. V. 62. P. 681.

[3] Yu.B. Gaididei, V. M. Loktev. Phys. Status Solidi B. 1988. V. 147. P. 307.

[4] F. C. Zhang, T. M. Rice. Phys. Rev. B. 1988. V. 37. P. 3759.

[5] V. J. Emery, G. Reiter. Phys. Rev. B. 1988. V. 38. P. 4547.

[6] B. Sriam Shastry. Phys. Rev. Lett. 1989. V. 63. P. 1288.

[7] F. C. Zhang. Phys. Rev. B. 1989. V. 39. P. 7375.

[8] D. M. Frenkel, R. J. Gooding, B. I. Shraiman, E. D. Siggia. Phys. Rev. B. 1990. V. 41. P. 350.

[9] J.L. Shen, C.S. Ting. Phys. Rev. B. 1990. V. 41. P. 1969.

[10] H. B. Pang, T. Xiang, Z. B. Su, L. Yu. Phys. Rev. B. 1990. V. 41. P. 7209.

[11] F. C. Zhang, T. M. Rice. Phys. Rev. B. 1990. V. 41. P. 7243.

[12] V. J. Emery, G. Reiter. Phys. Rev. B. 1990. V. 41. P. 7247.

[13] J. L. Shen, J. H. Xu, C. S. Ting, T. K. Lee. Phys. Rev. B. 1990. V. 42. P. 8728.

[14] P. W. Anderson. Science. 1987. V. 235. P. 1196.

[15] S. V. Lovtsov, V. Yu. Yushankhai. Physica C. 1991. V. 179. P. 159.

[16] S. V. Lovtsov, V. Yu. Yushankhai. Phys. Stat. Sol. (b). 1991. V. 166. P. 209.

[17] R. Hayn, V. Yu. Yushankhai, S. V. Lovtsov. Phys. Rev. B. 1993. V. 47. P. 5253.

[18] V. Yu. Yushankhai, V.S. Oudovenko, R. Hayn. Phys. Rev. B. 1997. V. 55. P. 15562.

[19] В. Ю. Юшанхай. Эффекты сильных электронных корреляций в моделях высокотемпературных сверхпроводников. Докторская диссертация. Дубна: ОИЯИ, 1999.

[20] J. H. Jefferson. Physica B. 1990. V. 165-166. P. 1013.

[21] J. H. Jefferson, H. Eskes, L.F. Feiner. Phys. Rev. B. 1992. V. 45. P. 7959.

[22] L. F. Feiner, J. H. Jefferson, R. Raimondi. Phys. Rev. B. 1996. V. 53. P. 8751.

[23] R. Raimondi, J. H. Jefferson, L. F. Feiner. Phys. Rev. B. 1996. V. 53. P. 8774.

[24] H.-B. Schuttler, A. J. Fedro. Phys. Rev. B. 1992. V. 45. P. 7588. 
[25] V.I. Belinicher, A. L. Chernyshev. Phys. Rev. B. 1993. V. 47. P. 390.

[26] V. I. Belinicher, A. L. Chernyshev. Physica C. 1993. V. 213. P. 298.

[27] V.I. Belinicher, A. L. Chernyshev. Phys. Rev. B. 1994. V. 49. P. 9746.

[28] V.I. Belinicher, A.L. Chernyshev, L. V. Popovici. Phys. Rev. B. 1994. V. 50. P. 13768; Phys. Rev. B. 1996. V. 53. P. 335.

[29] S. V. Lovtsov, N. M. Plakida, V. Yu. Yushankhai. Physica B. 1990. V. 165-166. P. 1007.

[30] N. M. Plakida, R. Hayn, J.-L. Richard. Phys. Rev. B. 1995. V. 51. P. 16599.

[31] N. M. Plakida. Physica C. 1997. V. 282-287. P. 1737.

[32] A. Fujimori, F. Minami. Phys. Rev. B. 1984. V. 30. P. 957.

[33] G. A. Sawatzky, J. W. Allen. Phys. Rev. Lett. 1984. V. 53. P. 2339.

[34] J. Zaanen, G. A. Sawatzky, J. M. Allen. Phys. Rev. Lett. 1985. V. 55. P. 418.

[35] F. Mila. Phys. Rev. B. 1988. V. 38. P. 11358.

[36] A. Fujimori. Phys. Rev. B. 1989. V. 39. P. 793.

[37] H. Eskes, G. A. Sawatzky, L.F. Feiner. Physica C. 1989. V. 160. P. 424.

[38] H. Eskes, L.H. Tjeng, G. A. Sawatzky. Phys. Rev. B. 1990. V. 41. P. 288.

[39] D. C. Mattis. Mod. Phys. Lett. B. 1994. V. 8. P. 1387.

[40] D. C. Mattis, J. M. Wheatley. Mod. Phys. Lett. 1995. V. 9. P. 1107.

[41] D. C. Mattis, J. M. Wheatley. Phys. Rev. B. 1995. V. 52. P. 15103.

[42] D. C. Mattis. Phys. Rev. Lett. 1995. V. 74. P. 3676.

[43] D. C. Mattis. Phys. Rev. B. 1999. V. 59. P. 7166.

[44] В. А. Москаленко, Н. Б. Перкинс. ТМФ. 1999. Т. 121. № 3. С. 464.

[45] V.A. Moskalenko, P. Entel, M. Marinaro, N. B. Perkins, C. Holfort. Phys. Rev. B. 2001. V. 63. P. 245119.

[46] Д. Ф. Дигор, П. Ентел, М. Маринаро, В.А. Москаленко, Н. Б. Перкинс. ТМФ. 2001. T. 127. № 2. С. 304 . 